\title{
The probability of particles screening on the screen, taking into account the interaction of particles over the meshes of the sieve
}

\author{
Aleksei Pelevin \\ U ral State M ining University, 620144, 30, K uibyshev st., Ekaterinburg, Russia
}

\begin{abstract}
The probability of a single particle screening through the mesh of a screen is usually estimated by the A.M. Goden formula. During screening along a screen sieve, a quantity of particles moves. Particles of different sizes interact with each other. Large particles block the meshes of the sieve and reduce the probability of small particles screening. A screening probability formula is proposed that takes into account the interaction of particles over the sieve meshes and the possibility of several particles passing simultaneously through the sieve mesh. The results of modeling the probability of sieving and screening process are presented. An increase in the fraction of "small" particles in the primary product leads to an increase in the probability of sieving. When the material moves along the screen sieve (from the first to the last row of meshes), the probability of particle sieving decreases.
\end{abstract}

Screening is widely used in ore crushing and concentration, in metallurgy, in the production of construction materials, and in other industries [1-3]. Screening rates depend on the amount of fine particles that have passed through the screening surface of the screen. Theoretical calculations of screening indicators are considered in many academic papers [4-6].

\section{Probability of a particle passing through a sieve mesh}

The probability of a particle passing through the meshes of the screening surface of the screen is determined by the A.M. Goden formula [7]:

$$
P=\varphi \cdot\left(\frac{a-d}{a}\right)^{\psi}=\varphi \cdot\left(1-\frac{d}{a}\right)^{\psi},
$$

where $\varphi$ - the effective screening area; $d$ - the particle size, $\mathrm{mm} ; a$ - the size of the sieve mesh, $\mathrm{mm} ; \Psi$ - coefficient taking into account the shape of the sieve mesh.

The probability of a particle passing through the screen sieve mesh depends on the difference between the size of the sieve mesh and particle. The probability of a particle passing through the sieve mesh decreases with particle size increasing. Formula (1) is used in many mathematical models to obtain the indicators of separation by size on the screen [8- 
11]. Formula (1) is valid for the case of sieving a single particle. During screening along a screen sieve, a quantity of particles moves. Particles of different sizes interact with each other. Large particles block the meshes of the sieve and reduce the probability of small particles screening. Therefore, the probability of particles sieving according to A.M. Goden model can be considered overpriced. A.M. Goden model reflects the general pattern of sieving particles of various sizes and is used for the entire length of the screen sieve. To take into account the design features of the screen and the granulometric size composition, correction factors are used.

\section{Probability of particle size range sieving}

In practice and in the modeling of the screening process, the size of the primary product is specified not by the sizes of single particles, but by the ranges of particle sizes. Therefore, for any class of particle size it is necessary to calculate the probability of sieving. The probability of the particle size range sieving can be found as the ratio of a certain integral of formula (1) to the range of particle size variation in the class [11]:

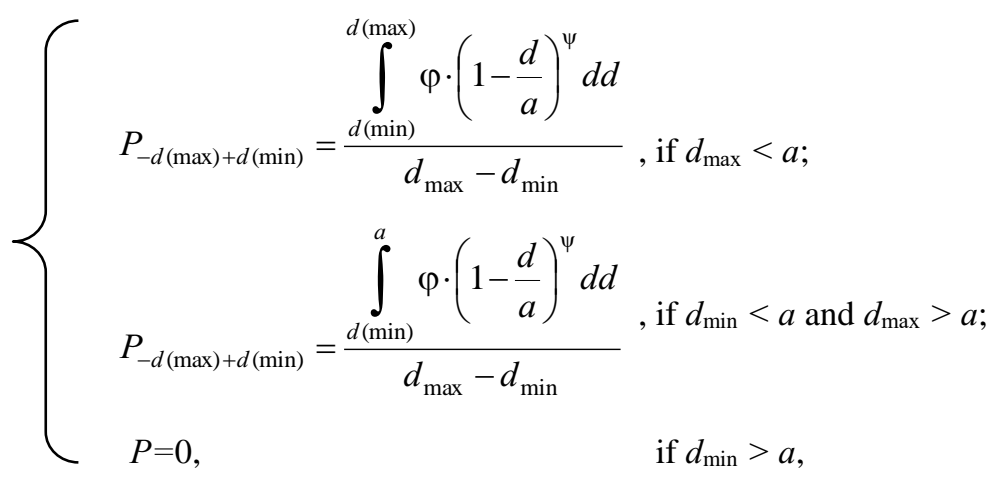

where $d_{\max }$ and $d_{\min }-$ maximum and minimum particle size in the range, $\mathrm{mm}$.

Final formulas for determining the probability of passing through the screen sieve of various particle size ranges:

- for needle-slot screens $(\Psi=1)$

$$
\left\{\begin{array}{cl}
P=\varphi \cdot \frac{\left(d_{\max }-d_{\min }\right)-\left(d_{\max }{ }^{2}-d_{\min }{ }^{2}\right) / 2 a}{\left(d_{\max }-d_{\min }\right)} & \text { if } d_{\max }<a ; \\
P=\varphi \cdot \frac{\left(a-d_{\min }\right)-\left(a^{2}-d_{\min }{ }^{2}\right) / 2 a}{\left(d_{\max }-d_{\min }\right)} & \text {, if } d_{\min }<a \text { and } d_{\max }>a ; \\
P=0 & \text {, if } d_{\min }>a
\end{array}\right.
$$

- for square-mesh sieve $(\Psi=2)$

$$
\left\{\begin{array}{cl}
P=\frac{\varphi \cdot a \cdot\left[\left(1-d_{\min } / a\right)^{3}-\left(1-d_{\max } / a\right)^{3}\right]}{3 \cdot\left(d_{\max }-d_{\min }\right)}, & \text { if } d_{\max }<a ; \\
P=\frac{\varphi \cdot a \cdot\left(1-d_{\min } / a\right)^{3}}{3 \cdot\left(d_{\max }-d_{\min }\right)}, & \text { if } d_{\min }<a \text { and } d_{\max }>a ; \\
P=0, & \text { if } d_{\min }>a,
\end{array}\right.
$$


Equations (2)-(4) are accepted as the basic equations included in the model of probability of particle sieving.

\section{Model for accounting the interaction of particles on the screen sieve}

For sieving particles with a size di through the sieve mesh, it is necessary that the particle is above the mesh. Using formulas (3) or (4) takes into account the probability of sieving particles of only one range of particle size. Particles of this size range will be prevented from appearing above the sieve mesh of a particle of a different size range. Large particles with a size larger than the sieve meshes will overlap the meshes of the screen sieve. Small particles of a different size range will be sieved, and occupy the volume of the sieve mesh. This is the process of particle interaction above the sieve mesh. Therefore, the probability of sieving particles of one particle size range will be affected by the amount in the primary product of particles with other particle size ranges. If the product enters the screening surface of the screen, the distribution of particles of different particle sizes over the height of the material on the sieve will be the same. Therefore, the probability of the appearance of particles of particle size range with a conditional size di above the screen sieve will be determined by the volume fraction of different particle size ranges on the screening surface of the screen [11].

The probability of the appearance of particles of particle size range with a conditional size di above the sieve mesh will be equal to:

$$
P_{0}\left(d_{i}\right)=\gamma\left(d_{i}\right)
$$

where $P_{0}\left(d_{i}\right)$ - the probability of the appearance of particles of size range with a conditional size $d_{i}$ above the sieve mesh; $\gamma\left(d_{i}\right)$ - the volume fraction of particles of the size range with a conditional size $d_{i}$ in the volume of the material above the sieve mesh; $i$ - the size range number.

Formula (5) is valid at the same density of all particles located on the screening surface of the screen.

The probability of passage of particles of size range with a conditional size $d_{i}$ through the sieve mesh, taking into account the interaction of particles of different size ranges over the meshes of the screening surface of the screen, equals to:

$$
P_{(-)}\left(d_{i}\right)=P_{0}\left(d_{i}\right) \cdot P^{a}\left(d_{i}\right)
$$

where $P^{a}\left(d_{i}\right)$ - the probability of sieving of particles of the size range through the sieve mesh, determined by the formulas (3) or (4).

\section{Model of the possibility of sieving through the sieve mesh of particles of several particle size ranges}

The probability $P_{(-)}\left(d_{i}\right)$ determines the possibility of sieving through the sieve mesh of particles of only one range of particle size. Therefore, the probability of passage of particles of the size range with a conditional size $d_{i}$ through the sieve of the screen $P_{(-)}\left(d_{i}\right)$ will be underestimated for small particles whose size is smaller than the size of the sieve cells. Particles of different particle sizes, which can simultaneously pass through the mesh, may appear above the sieve mesh. When sieving of a particle with a size $d_{l}$ through the sieve mesh 
of the screen, an unfilled part will appear in the sieve mesh through which other particles with sizes $d_{i}$ can pass. When sieving a fine particle of $d_{l}$ size through the sieve mesh, a conditional additional mesh $\left(a_{l}\right)$ is formed between the edge of this particle and the boundary of the sieve mesh with the size equal to: $a_{l}=a-d_{l}$ [11]. Other "small" particles of $d_{i}$ size can be sieved through this conditional additional mesh (Fig. 1).

The possibility of simultaneous passage of particles of different particle sizes through the meshes of the screening surface of the screen should be considered. Therefore, we can add an additional quantity $P^{a(l)}\left(d_{i}\right)$ in the formula (6):

$$
P_{(-)}\left(d_{i}\right)=P_{0}\left(d_{i}\right) \cdot P^{a}\left(d_{i}\right)+P^{a(l)}\left(d_{i}\right)
$$

where $P^{a}\left(d_{i}\right)$ - the probability of sieving particles of the size range through the sieve mesh (formulas (3) or (4)); $P^{a(l)}\left(d_{i}\right)$ - the probability of passage of particles of the particle size range with $d_{i}<a_{l}$ through the conditional additional mesh formed when a different size range of particles with size $d_{l}$ passes through the sieve mesh; $i$ - the number of the considered range of particle size; $l$ - the number of other particle size ranges of the particles forming the conditional meshes $a_{l}$.

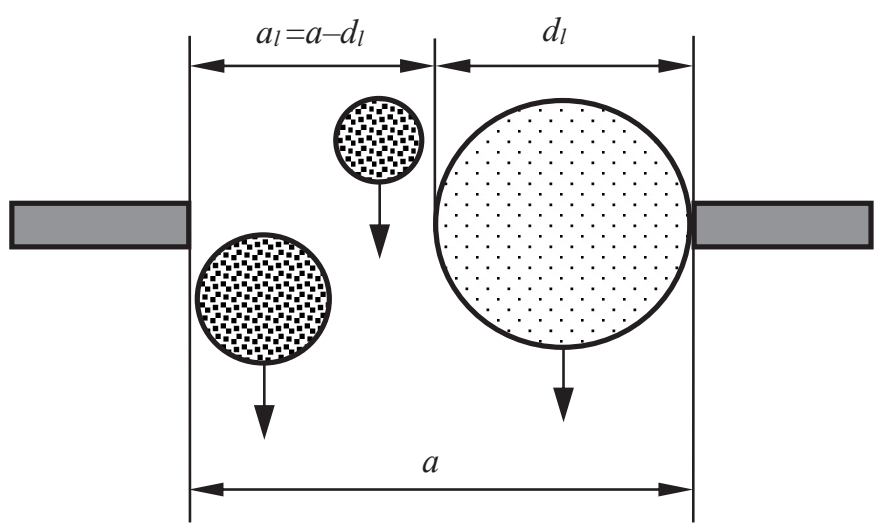

Fig. 1. Passing through the sieve mesh of the screen of several particles of various sizes.

The probability $P^{a(l)}\left(d_{i}\right)$ can occur when two conditions are satisfied.

1. The simultaneous appearance above the screen sieve of particles of two particle sizes with the nominal sizes $d_{i}$ and $d_{l}$, which can pass through the sieve mesh.

2. The simultaneous appearance in the screen sieve mesh of particles of two size ranges with nominal sizes $d_{i}$ and $d_{l}$.

When determining the value $P^{a(l)}\left(d_{i}\right)$, it is not necessary to take into account the coefficient of effective screening area. In this case, the conditional screening surface of the screen with conditional meshes is considered. The final formula for the probability of passage of particles of the size range with a conditional size $d_{i}$ through the screen sieve mesh will be as follows

$$
P_{(-)}\left(d_{i}\right)=P_{0}\left(d_{i}\right) \cdot P^{a}\left(d_{i}\right)+\sum_{l=1}^{n}\left(P_{0}\left(d_{i}\right) \cdot P_{0}\left(d_{l}\right) \cdot P^{a}\left(d_{l}\right) \cdot P^{(a-d(l))}\left(d_{i}\right)\right),
$$

where $P_{0}\left(d_{i}\right)$ and $P_{0}\left(d_{l}\right)$ - the probabilities of the appearance of particles with the particle size $d_{i}$ and $d_{l}$ above the sieve mesh, calculated by equation (5); $P^{a}\left(d_{l}\right)$ - the probability of 
sieving particles with the particle size $d_{l}$ through the sieve mesh, calculated by the formulas (3) or (4)); $P^{(a-d(l))}\left(d_{i}\right)$ - the probability of sieving the particles with the particle size $d_{i}$ through a conditional additional mesh formed when another particle with a particle size $d_{l}$ passes through the sieve mesh (calculated by formulas (3) or (4)); $n$ - the number of the size range, the particles of which, passing through the mesh of the screening surface of the screen, can form conditional additional meshes.

If the size of the particle under consideration $d_{i}$ is larger than the size of the conditional additional mesh $a_{l}=a-d_{l}$, then the probabilities $P^{(a-d(l))}\left(d_{i}\right)=0$ and $P^{a(l)}\left(d_{i}\right)=0$. In this case, the probability of particle sieving is calculated by the formula (6).

Formula (8) for calculating the probability of passing particles of a given size range with size $d_{i}$ through the screen sieve mesh takes into account two events.

1. The interaction of particles of different sizes above the sieve mesh (the probability of the appearance of particles of different sizes above the sieve mesh).

2. The interaction of particles of different sizes in the sieve mesh (the possibility of simultaneous passage of particles of different particle sizes through the mesh of the screening surface of the screen).

\section{Modeling the screening process}

The sieving probability is calculated for a screen with square sieve meshes with a size of 2 $\mathrm{mm}$. Modeling was performed for product No. 1 with a high proportion of "small" particles and for product No. 2 with a low proportion of "small" particles (Table 1). During the modelling, the process of segregation of particles on the sieve, the height of the material layer, and other factors were not taken into account.

Table 1. Granulometric composition of the primary products.

\begin{tabular}{|c|c|c|}
\hline \multirow{2}{*}{$\begin{array}{c}\text { Size class (range of } \\
\text { fineness), } \\
-d_{\max }+d_{\min }, \mathrm{mm}\end{array}$} & Size class mass content, \% \\
\cline { 2 - 3 } & Product N 0. 1 & Product N 0. 2 \\
$-5+4$ & - & 7 \\
$-3+3$ & 3 & 12 \\
\hline$-2+1.5$ & 9 & 17 \\
$-1.5+1$ & 20 & 11 \\
$-1+0.5$ & 25 & 12 \\
$-0.5+0$ & 31 & 24 \\
\hline Total & 100 & 100 \\
\hline
\end{tabular}

When using formula (8), it is necessary to determine the number and size of the conditional meshes $a_{l}=a-d_{l}$, formed when each particle size range passes through the sieve. During modeling, $d_{l}$ was equal to the maximum particle size in the size range. The possibility of simultaneously sieving of only two particles is considered. When sieving size classes $0.5+0,-1+0.5,-1.5+1 \mathrm{~mm}$, conditional additional meshes are formed: $a_{1}=2-0.5=1.5 \mathrm{~mm}$; $a_{2}=2-1=1 \mathrm{~mm} ; a_{3}=2-1.5=0.5 \mathrm{~mm}$. The size class $-2+1.5 \mathrm{~mm}$ and larger classes do not form additional conditional meshes.

Table 2 shows the probabilities of sieving particle size classes through the main square mesh of the screen sieve with $a=2 \mathrm{~mm}$ (formula (6)), through the conditional additional meshes $\left(P^{a(l)}\left(d_{i}\right)\right)$, and the total probability of sieving particle size classes for the first row of screen sieve meshes (formula (8)). Modeling of simultaneous sieving of several particles through the sieve mesh increases the probability of sieving. With an increase in the fraction of "small" particles in the primary product, the overall probability of the passage of "small" 
particles through the main mashes of the sieve $(a)$ and through additional conventional meshes $\left(a_{l}\right)$ increases.

Table 2. The probability of particles passing through the main and conditional meshes (first row of the screening surface meshes of the screen)

\begin{tabular}{|c|c|c|c|c|c|}
\hline $\begin{array}{c}\text { Size class, } \\
-d_{\max }+d_{\min }, \mathrm{mm}\end{array}$ & \multicolumn{6}{|c|}{ Probability of sieving through sieve meshes, \% } & $\begin{array}{c}\text { Total } \\
\text { probability, } \%\end{array}$ \\
\hline \multicolumn{7}{|c|}{} & $a=2 \mathrm{~mm}$ & \multicolumn{2}{c|}{$a_{1}=1.5 \mathrm{~mm}$} & \multicolumn{1}{c|}{$a_{2}=1 \mathrm{~mm}$} & $a_{3}=0.5 \mathrm{~mm}$ & \\
\hline$-2+1.5$ & 0.25 & - & - & - & 0.25 \\
\hline$-1.5+1$ & 2.92 & 0.18 & - & - & 3.10 \\
\hline$-1+0.5$ & 9.90 & 1.55 & - & - & 11.44 \\
\hline$-0.5+0$ & 23.90 & - & 1.79 & 0.30 & 25.99 \\
\hline \multicolumn{7}{|c|}{ Product N 0.1} \\
\hline$-2+1.5$ & 0.23 & - & - & - & 0.23 \\
\hline$-1.5+1$ & 1.75 & 0.08 & - & - & 1.83 \\
\hline$-1+0.5$ & 6.73 & 0.82 & - & - & 7.55 \\
\hline$-0.5+0$ & 18.50 & - & 0.94 & 0.14 & 19.58 \\
\hline
\end{tabular}

The particle size separation indicators depend on the length of the screening surface of the screen. The screening process was simulated on a screen sieve having 200 rows of meshes along the length of the screen at $\varphi=1$. This simulated the effect of the length of the screen sieve on the screening process. During the modeling, the process of segregation, the height of the material layer, and the features of moving the material along the screen were not taken into account. The change of the probability of particles screening and the output of the undersize product along the length of the screen is shown in Fig. 2 and in Fig. 3. When the material moves along the screen sieve (from the first to the last row of meshes), the probability of particle sieving decreases. On the initial section of the screen, the probability of sieving depends on the particle size and on the proportion of "small" particles in the primary product. The probability of particles sieving with a particle size of $-0.5+0 \mathrm{~mm}$ is higher than the probability of particles sieving with a particle size of $-1+0.5 \mathrm{~mm}$. The probability of particles sieving is lower for product N o. 2, compared with product No. 1. On the final section of the screen, the probability of sieving depends less on the particle size and on the proportion of "small" particles in the primary product (Fig. 2). In the final section of the screen, the proportion of "large" particles on the sieve increases. Large particles block the meshes of the sieve and reduce the probability of small particles screening.

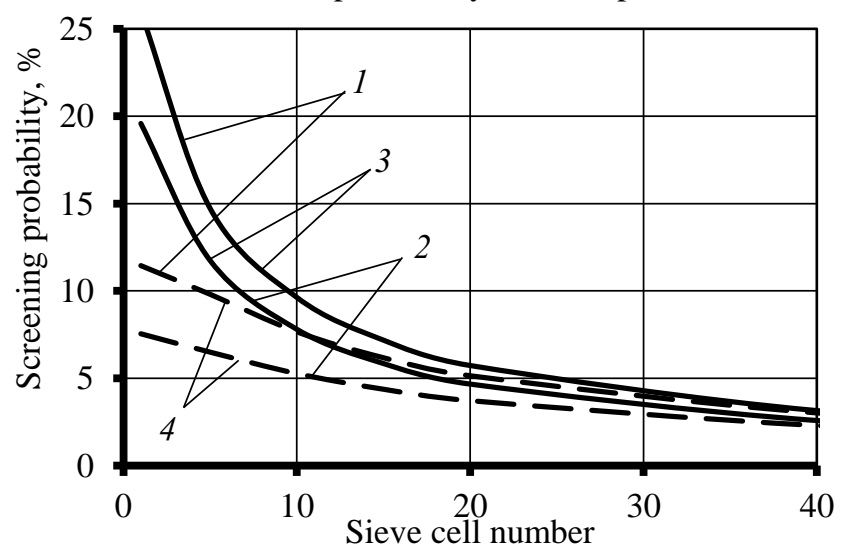

Fig. 2. Change of the probability of particles sieving along the length of the screen sieve: 1 - product No. $1 ; 2$ - product N $0.2 ; 3$ - class $-0.5+0 \mathrm{~mm} ; 4$ - class $-1+0.5 \mathrm{~mm}$. 


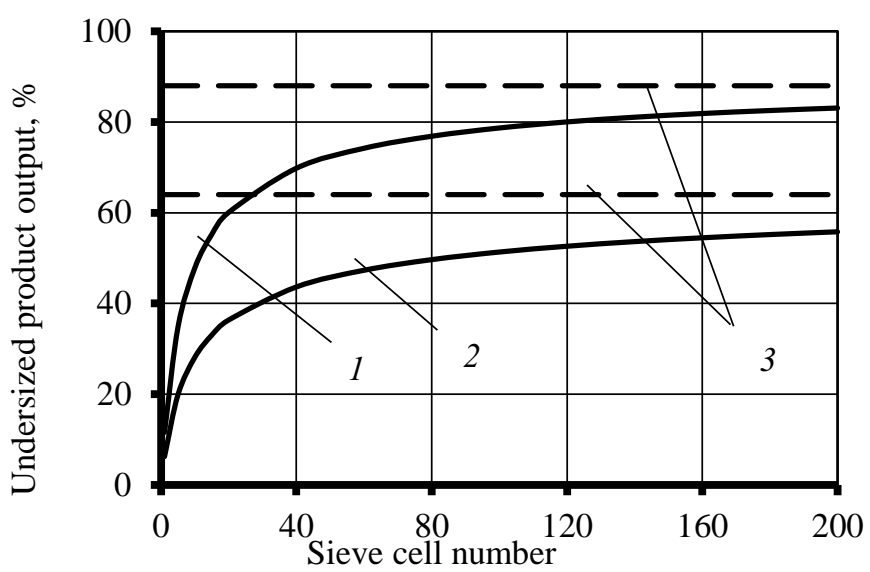

Fig. 3. Change in the output of the undersized product along the length of the screen: 1 - product No. 1; 2 - product No. 2; maximum output of the undersized product

W ith an increase in the length of the screen, the screening time increases. This leads to an increase in the output of the undersized product. With an increase in the duration of screening, the increase in the output of the undersized product decreases. W ith an increase in the share of "large" particles in the primary material, the output of the undersized product and other screening indicators decrease. Large particles block the sieve meshes and reduce the probability of small particles passing through the sieve meshes. Therefore, when screening products with a low proportion of "small" particles, it is necessary to use a screen with a larger sieve area (Fig. 3).

The modeling results reflect the general patterns of the screening process. Using the developed model of the probability of particles sieving allows us to evaluate the effect of the particle size distribution of the primary product on the screening process. The model of the probability of passing particles through the screen sieve meshes should take into account the mechanisms of interaction of particles of different sizes above the screen meshes and the possibility of simultaneous passage of particles of different sizes through the screen sieve mesh. In the considered model, the process of segregation of particles on the screen sieve is not taken into account.

\section{References}

1. S. Palaniandy, R. Halomoan, H. Ishikawa, Minerals Engineering, 133, 10 (2019)

2. A. E. Pelevin, N. A. Sytykh, Obogashchenie rud, 2, 13 (2018)

3. A. E. Pelevin, N. A. Sytykh, Obogashchenie rud, 4, 10 (2016)

4. M. Jahani, A. Farzanegan, M. Noaparast, Powder Technology, 283, 32 (2015)

5. F. Elskamp, H. Kruggel-Emden, Powder Technology, 26 (3), 679 (2015)

6. D. Markauskas, H. Kruggel-Emden, Advanced Powder Technology, 30(12), 2997 (2019)

7. A. M. Gaudin, Principles of mineral dressing, 432, (1932)

8. L. A. Vaisberg, K. S. Ivanov, T. N. Baldaeva, Obogashchenie rud, 1, 3 (2016)

9. L. A. Vaisberg, K. S. Ivanov, Construction materials, 2, 84 (2013)

10. L. A. Vaisberg, K. S. Ivanov, Solid municipal waste, 3, 22 (2013)

11. A. E. Pelevin, Izvestiya Vysshikh Uchebnykh Zavedenii. Gornyi Zhurnal, 1, 119 (2011) 